\title{
Derivation and Validation of a Risk Assessment Model for Immunomodulatory Drug-Associated Thrombosis Among Patients With Multiple Myeloma
}

Ang Li, MDa; Qian Wu, PhD ; Suhong Luo, MSc; Greg S. Warnickd; Neil A. Zakai, MDe; Edward N. Libby, MDf; Brian F. Gage, MD, MScg; David A. Garcia, MDa; Gary H. Lyman, MD, MPHd,f; and Kristen M. Sanfilippo, MD, MPHSc,g

\section{ABSTRACT}

Background: Although venous thromboembolism (VTE) is a significant complication for patients with multiple myeloma (MM) receiving immunomodulatory drugs (IMiDs), no validated clinical model predicts VTE in this population. This study aimed to derive and validate a new risk assessment model (RAM) for IMiD-associated VTE. Methods: Patients with newly diagnosed MM receiving IMiDs were selected from the SEER-Medicare database $(n=2,397)$ to derive a RAM and then data from the Veterans Health Administration database $(n=1,251)$ were used to externally validate the model. A multivariable causespecific Cox regression model was used for model development. Results: The final RAM, named the "SAVED" score, included 5 clinical variables: prior surgery, Asian race, VTE history, age $\geq 80$ years, and dexamethasone dose. The model stratified approximately $30 \%$ of patients in both the derivation and the validation cohorts as high-risk. Hazard ratios (HRs) were $1.85(P<.01)$ and $1.98(P<.01)$ for highversus low-risk groups in the derivation and validation cohorts, respectively. In contrast, the method of stratification recommended in the current NCCN Guidelines for Cancer-Associated Venous Thromboembolic Disease had HRs of $1.21(P=.17)$ and $1.41(P=.07)$ for the corresponding risk groups in the 2 datasets. Conclusions: The SAVED score outperformed the current NCCN Guidelines in risk-stratification of patients with $\mathrm{MM}$ receiving IMiD therapy. This clinical model can help inform providers and patients of VTE risk before IMiD initiation and provides a simplified clinical backbone for further prognostic biomarker development in this population.

J Natl Compr Canc Netw 2019;17(7):840-847 doi: 10.6004/jnccn.2018.7273

\footnotetext{
${ }^{a}$ Division of Hematology, University of Washington School of Medicine, and ${ }^{b}$ Clinical Research Division, Fred Hutchinson Cancer Research Center, Seattle, Washington; 'Research Service, St. Louis Veterans Affairs Medical Center, St. Louis, Missouri; ${ }^{d}$ Public Health Sciences Division, Fred Hutchinson Cancer Research Center, Seattle, Washington; ${ }^{e}$ Departments of Medicine and Pathology and Laboratory Medicine, Larner College of Medicine at the University of Vermont, Burlington, Vermont; 'Division of Medical Oncology, University of Washington School of Medicine, Seattle, Washington; and 9Division of General Medical Sciences, Washington University School of Medicine, St. Louis, Missouri.
}

\section{Background}

Multiple myeloma (MM) is associated with a high risk of venous thromboembolism (VTE), especially when using treatment regimens containing immunomodulatory drugs (IMiDs; eg, lenalidomide or thalidomide). ${ }^{1}$ Initial trials of IMiDs without pharmacologic VTE prophylaxis reported a VTE rate of 4.1 per 100 patient-months. ${ }^{2}$ To prevent IMiDassociated VTE in patients with MM, experts from the International Myeloma Working Group (IMWG) proposed a VTE risk assessment consensus guide that incorporated individual, myeloma-related, and therapy-related risk factors, ${ }^{3}$ and recommended prophylactic anticoagulation for patients with $\geq 2$ of these risk factors. The same consensus was later incorporated into the NCCN Clinical Practice Guidelines in Oncology (NCCN Guidelines) for CancerAssociated Venous Thromboembolic Disease and became the cornerstone for VTE risk stratification in subsequent MM clinical trials. ${ }^{4}$ However, adherence to these guidelines has been poor outside of clinical studies, ${ }^{5,6}$ and their performance has never been validated in an independent population. ${ }^{7,8}$

To address these issues, we conducted a large population-based cohort study using the SEER-Medicare and Veterans Health Administration (VHA) databases. We assessed the performance of the current NCCN Guidelines in each dataset and further developed and validated an evidence-based VTE risk assessment model (RAM) for patients with MM receiving IMiDs.

\section{Methods}

\section{Derivation Cohort Selection}

A retrospective cohort study was conducted using the SEERMedicare database as the derivation cohort. ${ }^{9}$ Patients were included if they were aged $>65$ years with newly diagnosed MM in January 2007 through December 2013 and had

\section{See JNCCN.org for supplemental online content.}


a prescription for IMiDs within 12 months of diagnosis, complete enrollment in fee-for-service plans for least 12 months (last available claims in December 2014), and prescription drug coverage for at least 6 months before the IMiD index date. Patients were excluded if they had alternative Medicare eligibility, such as end-stage renal disease or disability, or a recent diagnosis of VTE within 6 months of first IMiD prescription (Figure 1).

\section{Outcome and Risk Factor Ascertainment}

For outcome and risk factor ascertainment, administrative claims codes (ICD-9-CM, Current Procedural Terminology, version 4 [CPT4], Health Care Procedure Classification Code [HCPCS], and National Drug Code [NDC]) were obtained from Medicare Provider Analysis and Review (MEDPAR), carrier claims (ie, National Claims History), outpatient claims, and durable medical equipment files. Generic drug names for oral medications were obtained from Medicare Part D Prescription Drug
Event files (see supplemental eTable 1, available with this article at JNCCN.org). The primary VTE outcome was defined as either 1 inpatient or 2 outpatient claims at least 30 days apart in combination with an anticoagulant prescription within 90 days. ${ }^{10}$ The ICD-9-CM codes used in this definition included pulmonary embolism (PE), lower extremity deep vein thrombosis (LE-DVT), and upper extremity DVT (UE-DVT) and had a positive predictive value of $75 \%$ to $95 \%$ based on clinical record review (supplemental eTable 2). ${ }^{11}$ Patients were observed from 7 days after first IMiD prescription until first VTE occurrence or death. Individuals were censored at disenrollment from Medicare Part A, B, or D; enrollment in an HMO; 90 days after discontinuation of IMiD; or December 2014, whichever came first.

Demographics information was obtained from the Patient Entitlement and Diagnosis Summary File. For this study, race was classified as white, black, or Asian (including Asian, Pacific Islander, American Indian, and

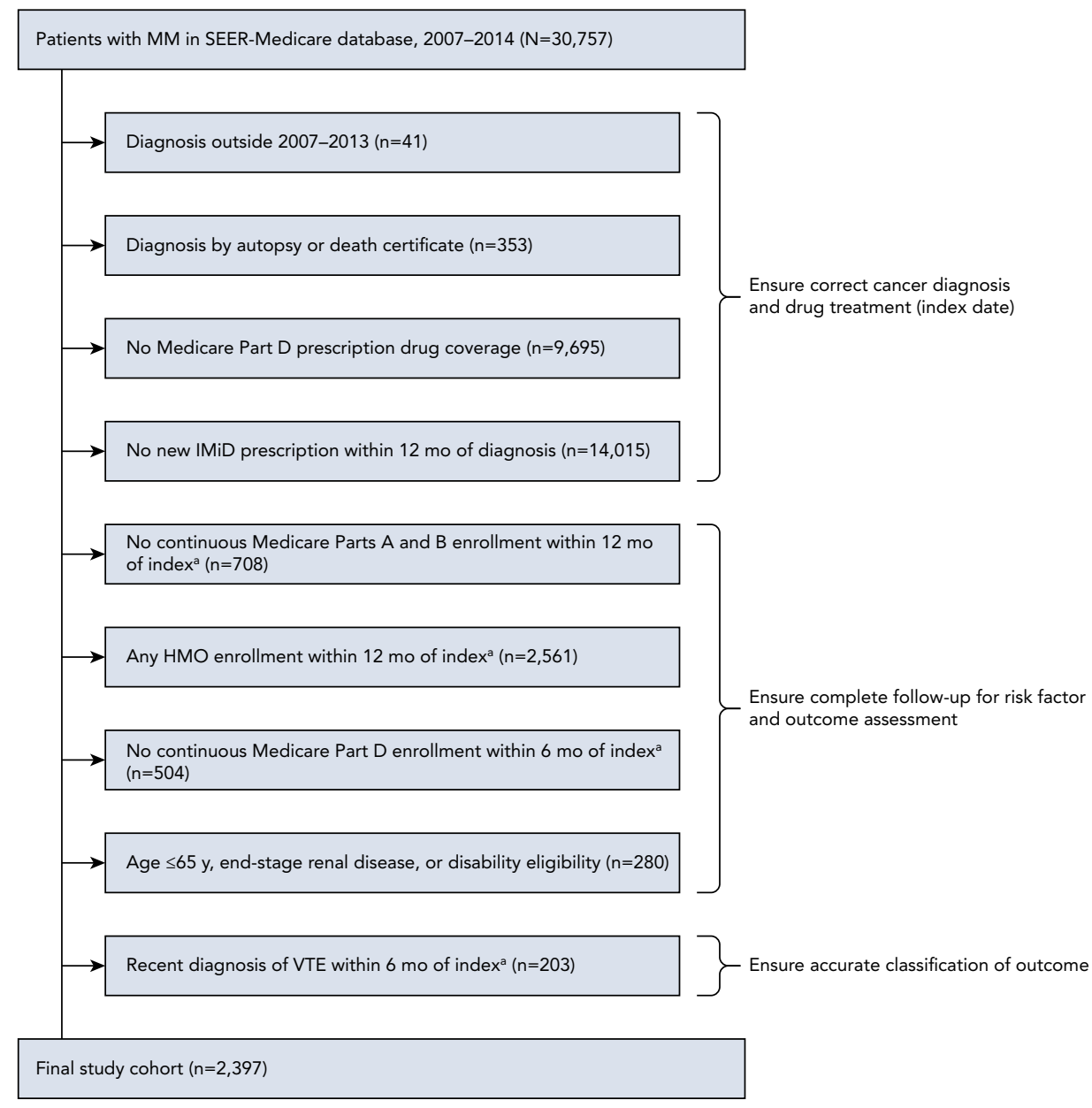

Figure 1. Derivation cohort patient selection and exclusion schema.

Abbreviations: IMiD, immunomodulatory drug; MM, multiple myeloma; VTE, venous thromboembolism.

alndex date was determined by date of first new IMiD prescription (must be within 12 months of initial MM diagnosis) and ranged from January 1, 2007-June 31, 2014, to ensure that patients had at least 6 months of follow-up (unless censored by death or disenrollment) because Medicare claims ended on December 31,2014 . 
Alaskan Native) according to SEER race recode. VTE risk factors including obesity, congestive heart failure, myocardial infarction, cardiac arrhythmias, diabetes, hypertension, renal disease, liver disease, immobilization (paralysis/hemiplegia), blood clotting disorders (coagulopathy), anemia, history of VTE, history of bleeding, central venous catheter, recent sepsis, surgery, and traumatic injury were ascertained from patient Medicare files (supplemental eTable 2). ${ }^{12-15}$ Concurrent chemotherapy and erythropoietin were defined using a 30-day window before and after an IMiD prescription (supplemental eTable 3). ${ }^{16}$ Dexamethasone dose per cycle was calculated from the average monthly dexamethasone dose over 30 days, with low dose defined as $<120 \mathrm{mg}$, standard dose as 120 to $160 \mathrm{mg}$, and high dose as $>160 \mathrm{mg}$ per cycle. Concurrent anticoagulants were defined by any anticoagulant prescription written within 30 days before to 7 days after the date of IMiD prescription.

\section{Validation Cohort Selection}

External validation was performed using a separate cohort of patients from the VHA database that linked the VA Central Cancer Registry and the VHA electronic medical record. Veteran patients aged $\geq 65$ years with newly diagnosed MM who were prescribed an IMiD within 12 months of diagnosis but did not have a recent VTE were identified using a patient selection algorithm similar to the one shown in Figure 1. A sensitivity analysis was performed using patients of all ages. Covariates from the derivation process were defined using similar claims codes as described in the previous section using the VA Informatics and Computing Infrastructure platform. All drug administration was captured using pharmacy benefits management records. The VTE outcome up to 1 year after diagnosis was obtained and adjudicated by the last author (K.M. Sanfilippo).

\section{Statistical Analysis}

To evaluate the performance of the NCCN Guidelines for Cancer-Associated Venous Thromboembolic Disease, we followed the risk stratification scheme from the January 2018 version of the guidelines. ${ }^{4}$ Patients were assigned to the high-risk group if they had any of the following therapy-related risk factors: dexamethasone $\geq 480 \mathrm{mg}$, doxorubicin, or multiagent chemotherapy, or if they had $\geq 2$ of the following individual risk factors: prior VTE, obesity, central venous catheter or pacemaker, cardiac disease (myocardial infarction, congestive heart failure, or cardiac arrhythmias), chronic renal disease, diabetes, acute infection (sepsis), paralysis/hemiplegia, recent general surgery or anesthesia, traumatic injury, erythropoietin treatment, or coagulopathy. Discrimination of the NCCN risk group was assessed by the hazard ratio (HR) and Harrell's c-index from the Cox regression model.
To derive a new RAM, we first examined the association between VTE and an expanded set of risk factors in univariate Cox regression models (supplemental eTable 4). We then used a backward stepwise algorithm with a cutoff $P$ value of .15 for variable selection in the multivariable Cox regression model. Continuous variables in the final model (eg, age) were dichotomized according to the optimized c-index. Variables from the Cox regression model were incorporated into a RAM where integer scores were assigned according to the $\beta$ coefficients. The model's discrimination was internally and externally validated using Harrell's c-indices with 200 bootstrap samples for the $95 \%$ CI. ${ }^{17}$ Risk group performance was evaluated by HR and $P$ value. The RAM was calibrated by comparing the Kaplan-Meier failure estimates in the 2 datasets. ${ }^{8}$ Interactions were tested using the likelihood ratio test. Proportionality assumption was tested using the Schoenfeld residuals. Analyses were performed using STATA 14.2 (StataCorp LP), R-3.5.1 (R Foundation), and SAS 9.4 (SAS Institute Inc.).

This research was deemed exempt by the University of Washington's Institutional Review Board (IRB) and conformed to all principles of the Declaration of Helsinki. Use of the VHA database was approved by the St. Louis Veterans Affairs Medical Center IRB.

\section{Results}

\section{Cohort Descriptions}

Baseline patient characteristics for the derivation (SEERMedicare) and external validation (VHA) cohorts are shown in Table 1. A total of 2,397 patients with MM from the SEER-Medicare database met the study inclusion and exclusion criteria (Figure 1). Comparison of the demographics of the final study cohort and the overall MM cohort is shown in supplemental eTable 5. The differences seen with age and year of diagnosis were related to the nature of the cohort design and reflected the increasing enrollment in Medicare Part D during the 7-year period. ${ }^{18}$ In the derivation cohort, median age was 74 years (interquartile range [IQR], 70-80 years), $49 \%$ were female, and $80 \%$ were white, $13 \%$ were black, and $7 \%$ were Asian (Table 1). More patients received lenalidomide (78\%) than thalidomide (22\%). Median time from diagnosis to IMiD initiation was 59 days (IQR, 37-135 days) and median time on IMiD treatment was 116 days (IQR, 28-279 days). Concurrent treatments included dexamethasone in $60 \%$ of patients and bortezomib in $20 \%$. Only $13 \%$ of patients had anticoagulant exposure $(11 \%$ warfarin, $1 \%$ lowmolecular-weight heparin [LMWH], $1 \%$ direct oral anticoagulant [DOAC]), with a median duration of 116 days (IQR, $42-315$ days). VTE occurred in 346 patients (14.4\%), with 200 events (8.3\%) occurring during the first 12 months of continuous IMiD exposure. Specifically, there were 90 PEs, 196 LE-DVTs, and 60 UE-DVTs. Incidence of 


\section{Table 1. Baseline Characteristics for Derivation} and External Validation Cohorts

\begin{tabular}{|c|c|c|}
\hline & $\begin{array}{l}\text { Derivation Cohort } \\
\text { (SEER-Medicare; } \\
\mathbf{N}=\mathbf{2 , 3 9 7 )} \\
\%(\mathbf{n})\end{array}$ & $\begin{array}{c}\text { External Validation } \\
\text { Cohort (VHA; } \\
N=1,251) \\
\%(n)\end{array}$ \\
\hline Age (IQR), y & $74(70-80)$ & $74(69-79)$ \\
\hline Female sex & $49 \%(1,181)$ & $2 \%(22)$ \\
\hline \multicolumn{3}{|l|}{ Race } \\
\hline White & $80 \%(1,909)$ & $76 \%(949)$ \\
\hline Black & $13 \%(303)$ & $22 \%(273)$ \\
\hline Asian & $7 \%(159)$ & $2 \%(29)$ \\
\hline Unknown/Missing & $1 \%(26)$ & $0 \%(0)$ \\
\hline \multicolumn{3}{|l|}{ Year of diagnosis } \\
\hline$<2007$ & $0 \%$ & $37 \%(461)$ \\
\hline 2007 & $8 \%(202)$ & $10 \%(131)$ \\
\hline 2008 & $13 \%(311)$ & $11 \%(133)$ \\
\hline 2009 & $12 \%(294)$ & $11 \%(136)$ \\
\hline 2010 & $14 \%(341)$ & $8 \%(96)$ \\
\hline 2011 & $15 \%(362)$ & $8 \%(105)$ \\
\hline 2012 & $18 \%(419)$ & $8 \%(103)$ \\
\hline 2013 & $20 \%(468)$ & $7 \%(86)$ \\
\hline \multicolumn{3}{|l|}{ IMiD type } \\
\hline Lenalidomide & $78 \%(1,863)$ & $40 \%(497)$ \\
\hline Thalidomide & $22 \%(534)$ & $60 \%(754)$ \\
\hline \multicolumn{3}{|l|}{ Relevant comorbidities } \\
\hline $\mathrm{CHF}$ & $15 \%(360)$ & $12 \%(147)$ \\
\hline MI & $5 \%(116)$ & $4 \%(49)$ \\
\hline CA & $19 \%(446)$ & $15 \%(191)$ \\
\hline Obesity & $6 \%(137)$ & $26 \%(330)$ \\
\hline Diabetes & $27 \%(649)$ & $30 \%(376)$ \\
\hline Hypertension & $69 \%(1,641)$ & $75 \%(937)$ \\
\hline Renal disease & $27 \%(636)$ & $23 \%(290)$ \\
\hline Liver disease & $1 \%(31)$ & $2 \%(26)$ \\
\hline Paralysis/Hemiplegia & $1 \%(31)$ & $1 \%(16)$ \\
\hline Coagulopathy & $12 \%(277)$ & $7 \%(92)$ \\
\hline Anemia & $53 \%(1,273)$ & $52 \%(650)$ \\
\hline History of VTE & $2 \%(45)$ & $1 \%(14)$ \\
\hline History of bleeding & $8 \%(181)$ & $3 \%(38)$ \\
\hline Central venous catheter & $1 \%(35)$ & $1 \%(14)$ \\
\hline Recent sepsis & $5 \%(121)$ & $1 \%(16)$ \\
\hline Recent surgery & $2 \%(44)$ & $3 \%(32)$ \\
\hline Recent traumatic injury & $16 \%(373)$ & $8 \%(102)$ \\
\hline Recent erythropoietin & $12 \%(290)$ & $20 \%(245)$ \\
\hline
\end{tabular}

(continued)

Abbreviations: $\mathrm{CA}$, cardiac arrhythmia; $\mathrm{CHF}$, congestive heart failure; IMiD, immunomodulatory drug; IQR, interquartile range; $\mathrm{LMWH}$, low-molecularweight heparin; MI, myocardial infarction; VHA, Veterans Health Administration; VTE, venous thromboembolism.

\begin{tabular}{|c|c|c|}
\hline & $\begin{array}{l}\text { Derivation Cohort } \\
\text { (SEER-Medicare; } \\
\mathbf{N}=2,397) \\
\%(n)\end{array}$ & $\begin{array}{c}\text { External Validation } \\
\text { Cohort (VHA; } \\
N=1,251) \\
\%(n)\end{array}$ \\
\hline \multicolumn{3}{|l|}{ Concurrent chemotherapy } \\
\hline Bortezomib & $20 \%(486)$ & $11 \%(141)$ \\
\hline Melphalan & $1 \%(15)$ & $15 \%(184)$ \\
\hline Cyclophosphamide & $1 \%(27)$ & $1 \%(15)$ \\
\hline Doxorubicin & $1 \%(17)$ & $1 \%(9)$ \\
\hline \multicolumn{3}{|l|}{ Concurrent dexamethasone } \\
\hline $\begin{array}{l}\text { None or low dose } \\
(<120 \mathrm{mg} / \text { cycle })\end{array}$ & $41 \%(972)$ & $31 \%(387)$ \\
\hline $\begin{array}{l}\text { Standard dose } \\
(120-160 \mathrm{mg} / \text { cycle })\end{array}$ & $40 \%(952)$ & $49 \%(616)$ \\
\hline $\begin{array}{l}\text { High dose } \\
\text { (>160 mg/cycle) }\end{array}$ & $20 \%(473)$ & $20 \%(248)$ \\
\hline \multicolumn{3}{|l|}{ Concurrent anticoagulant } \\
\hline None & $87 \%(2,097)$ & $79 \%(991)$ \\
\hline Warfarin & $11 \%(252)$ & $16 \%(195)$ \\
\hline LMWH & $1 \%(35)$ & $5 \%(57)$ \\
\hline Direct oral anticoagulant & $1 \%(13)$ & $1 \%(8)$ \\
\hline
\end{tabular}

Abbreviations: $\mathrm{CA}$, cardiac arrhythmia; $\mathrm{CHF}$, congestive heart failure; IMiD, immunomodulatory drug; IQR, interquartile range; LMWH, low-molecularweight heparin; MI, myocardial infarction; VHA, Veterans Health Administration; VTE, venous thromboembolism.

VTE was $5.1 \%$ at 3 months, $8.7 \%$ at 6 months, and $12.5 \%$ at 12 months after IMiD initiation.

The external validation cohort consisted of 1,251 patients with MM (Table 1). The distribution of age and comorbidity was similar in both groups. However, in contrast to the SEER-Medicare cohort, patients in the VHA cohort were predominantly male $(98 \%)$, with a higher proportion of black patients (22\%) and a lower proportion of Asian patients (2\%). As the validation cohort predated 2007, there was more thalidomide $(60 \%)$ and melphalan (15\%) use and less lenalidomide $(40 \%)$ and bortezomib (11\%) use; otherwise, dexamethasone (69\%) and anticoagulant administrations $(21 \%)$ were similar in both cohorts. VTE occurred in 117 patients $(9.4 \%)$ within 12 months of IMiD initiation.

\section{Assessment of NCCN Guidelines Performance}

We assessed the performance of the NCCN Guidelines for Cancer-Associated Venous Thromboembolic Disease in both datasets (Table 2). Among 2,397 patients with MM in the derivation cohort, the guideline stratified 1,023 patients (43\%) into a high-risk group and 1,374 (57\%) into a low-risk group. Incidence of VTE at 3 and 6 months was $5 \%$ and $9 \%$, respectively, in the high-risk group, and $5 \%$ and $8 \%$, respectively, in the low-risk group (HR, 1.21; $P=.17$; $\mathrm{c}$-index $=0.51$ ) (Figure 2). In the sensitivity analysis, from which patients being treated with previous anticoagulants $(n=300)$ were 
Table 2. Univariate Cox Regression Analysis of NCCN Guideline Performance

\begin{tabular}{|c|c|c|c|c|}
\hline \multirow[b]{2}{*}{ Proposed Risk Factor } & \multicolumn{2}{|c|}{$\begin{array}{l}\text { SEER-Medicare } \\
\text { (Derivation Cohort) }\end{array}$} & \multicolumn{2}{|c|}{$\begin{array}{c}\text { VHA } \\
\text { (External Validation Cohort) }\end{array}$} \\
\hline & $\mathrm{HR}^{\mathrm{a}}$ & P Value & $\mathrm{HR}^{\mathrm{a}}$ & $P$ Value ${ }^{a}$ \\
\hline \multicolumn{5}{|l|}{ Individual } \\
\hline Prior VTE & 3.20 & $<.01$ & 3.85 & $<.01$ \\
\hline Obesity & 0.86 & .63 & 1.26 & .24 \\
\hline Chronic renal disease & 1.14 & .38 & 1.09 & .75 \\
\hline Diabetes & 1.14 & .38 & 0.76 & .23 \\
\hline Acute infection & 1.42 & .20 & 1.60 & .64 \\
\hline Immobilization & 1.24 & .71 & NE & N/A \\
\hline Blood clotting disorders & 0.96 & .86 & 1.67 & .31 \\
\hline \multicolumn{5}{|l|}{ Myeloma-related } \\
\hline Diagnosis of myeloma per se $\mathrm{b}^{\mathrm{b}}$ & N/A & N/A & N/A & N/A \\
\hline Hyperviscosity ${ }^{c}$ & N/A & N/A & N/A & N/A \\
\hline \multicolumn{5}{|l|}{ Therapy-related } \\
\hline High-dose dexamethasone, $\geq 480 \mathrm{mg}$ & 1.09 & .55 & 1.89 & $<.01$ \\
\hline Doxorubicin & NE & N/A & NE & N/A \\
\hline Multiagent (cytotoxic) chemotherapy & 2.05 & .48 & 0.91 & .77 \\
\hline Risk stratification $^{d}$ & \multicolumn{2}{|c|}{ HR (high/low) $=1.21(P=.17)$} & \multicolumn{2}{|c|}{ HR (high/low) $=1.41(P=.07)$} \\
\hline
\end{tabular}

Abbreviations: CA, cardiac arrhythmia; CHF, congestive heart failure; HR, hazard ratio; MI, myocardial infarction; N/A, not applicable; NE, no event; VHA, Veterans Health Administration; VTE, venous thromboembolism.

aHRs and $P$ values were estimated from univariate Cox regression analyses because risk factors from the guidelines were not derived statistically.

bDiagnosis of myeloma per se was not assigned any point because all patients had a myeloma diagnosis.

cHyperviscosity was a clinical definition that could not be defined accurately using claims data.

d"High-risk" was defined as $\geq 2$ risk factors in the individual or myeloma-related categories or any factor in the therapy-related category according to the NCCN Guidelines. The HR and Harrell's c-index were directly estimated from the regression of the final risk groups.

excluded, the resulting model had an HR of $1.34(P=.04)$ for high- versus low-risk groups, with a c-index of 0.52 .

The analysis was repeated using the independent VHA cohort (Table 2 and Figure 2). The NCCN Guidelines similarly did not accurately predict early onset of VTE, with an incidence of $5 \%$ and $9 \%$ in the high-risk group and $4 \%$ and $8 \%$ in the low-risk group at 3 and 6 months, respectively ( $\mathrm{HR}, 1.41 ; P=.07$; $\mathrm{c}$-index $=0.54$ ). In a sensitivity analysis that included patients of all ages, the risk stratification had a similar magnitude of discrimination but had more precision (HR, 1.42; $P=.01$; c-index $=0.54$ ).

\section{Derivation and Validation of the New RAM}

(SAVED Score)

Univariate analysis for all potential risk factors in the derivation cohort is shown in supplemental eTable 4. Five independent predictors of VTE were identified through multivariable regression (Table 3 ). Weighting each variable according to its beta coefficient, we created an ordinal score from -3 to +8 . Variables in the final model included (with point assignment) surgery within 90 days $(+2)$, Asian race $(-3)$, VTE history $(+3)$, age $\geq 80$ years $(+1)$, and dexamethasone dose ( +2 for high, +1 for standard), the sum of which represented the "SAVED" score.

To identify patients at the highest risk for VTE that would warrant thromboprophylaxis, we further stratified the SAVED score into 2 risk groups. In the derivation cohort, 686 patients $(29 \%)$ with a score $\geq 2$ were considered high-risk, and 1,711 patients $(71 \%)$ with a score $\leq 1$ were considered low-risk. Incidence of VTE at 3 and 6 months was $7 \%$ and $12 \%$ in the high-risk group, respectively, versus $4 \%$ and $7 \%$ in the low-risk group, respectively (Figure 3). The HR for VTE was $1.85(P<.01)$ with a c-index of $0.61(0.57-0.64)$ (Table 3$)$.

The RAM was externally validated using the VHA dataset (validation cohort; Table 3). The same risk predictors had a similar direction and magnitude in both datasets with the 


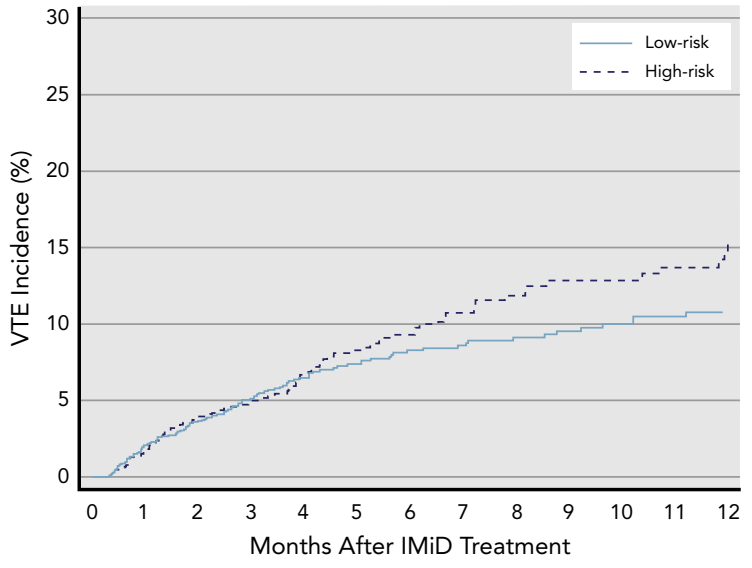

\begin{tabular}{|c|c|c|c|c|c|}
\hline & \multirow[b]{2}{*}{ HR } & \multirow[b]{2}{*}{ Risk Stratification } & \multicolumn{3}{|c|}{ VTE Incidence $(95 \% \mathrm{Cl})$} \\
\hline & & & $3 \mathrm{mo}$ & $6 \mathrm{mo}$ & $12 \mathrm{mo}$ \\
\hline \multirow{2}{*}{$\begin{array}{l}\text { SEER- } \\
\text { Medicare }\end{array}$} & \multirow[t]{2}{*}{$1.21(P=.17)$} & $\operatorname{High}(n=1,023)$ & $5 \%(4-7)$ & $9 \%(7-12)$ & $15 \%(12-19)$ \\
\hline & & Low $(n=1,374)$ & $5 \%(4-6)$ & $8 \%(7-10)$ & $11 \%(9-13)$ \\
\hline \multirow[t]{2}{*}{ VHA } & \multirow[t]{2}{*}{$1.41(P=.07)$} & High $(n=587)$ & $5 \%(4-7)$ & $9 \%(7-12)$ & $13 \%(10-16)$ \\
\hline & & Low $(n=664)$ & $4 \%(3-6)$ & $8 \%(6-10)$ & $9 \%(7-11)$ \\
\hline
\end{tabular}

Figure 2. VTE incidence and Kaplan-Meier failure curve of patients stratified by NCCN Guidelines.

Abbreviations: $\mathrm{HR}$, hazard ratio; IMiD, immunomodulatory drug; VHA, Veterans Health Administration; VTE, venous thromboembolism.

exception of older age. The SAVED model stratified 414 patients (33\%) as high-risk and 837 patients $(67 \%)$ as lowrisk. The corresponding HR for VTE was $1.98(P<.01)$ in the high-risk versus low-risk strata with a c-index of 0.60 (0.54-0.64). The new model calibrated well with similar expected and observed VTE events at different time points (Figure 3).

We performed 3 sensitivity analyses using the derivation cohort and 1 sensitivity analysis using the validation cohort. First, we examined the performance of the model in the derivation cohort after removing patients who had baseline anticoagulation for any reason. Using the subcohort of 2,097 patients without anticoagulant exposure, the resulting c-index was 0.60 and HR was $1.68(P<.01)$ for the high-risk versus the low-risk group. Second, we used a more restrictive definition for VTE (inpatient diagnosis of VTE only) in the derivation cohort; the corresponding c-index was 0.62 and the HR for VTE was $1.97(P<.01)$ between the high-risk versus the low-risk group. Third, we examined $\mathrm{PE}$ and/or LE-DVT as the outcome after excluding UEDVT and found that the SAVED RAM had a c-index of 0.58 and an HR of $1.96(P<.01)$ for the 2 risk groups. Finally, we included patients of all ages in the validation cohort rather than restricting the patients to those aged $>65$ years. In this expanded analysis of 2,208 Veterans Affairs patients, the SAVED RAM retained a c-index of 0.58 and a corresponding HR of $1.83(P<.01)$ between the risk groups.

\section{Discussion}

With improved MM-directed and supportive care, VTE has emerged as a significant treatment-associated complication of IMiD therapy. ${ }^{2,3}$ Using 2 nationwide databases with $>3,600$ patients, we derived and externally validated a simple RAM that identifies patients with a 2-fold increased risk for IMiD-associated VTE. It is the first validated clinical RAM for patients with MM receiving an IMiD.

The new SAVED model, with only 5 variables, has greater discriminative power than the more complex consensus currently recommended by the NCCN Guidelines for Cancer-Associated Venous Thromboembolic Disease. This improvement is likely related to several factors. First, we included demographics such as age and race. Second, we assigned differential weighting with more points to stronger predictive factors. Third, we used a modern dataset

\section{Table 3. Derivation and Validation of SAVED Score Using Multivariable Cox Regression Analysis}

\begin{tabular}{|c|c|c|c|c|c|}
\hline \multirow[b]{2}{*}{ Variable } & \multicolumn{2}{|c|}{$\begin{array}{l}\text { Derivation Cohort } \\
\text { (SEER-Medicare) }\end{array}$} & \multicolumn{2}{|c|}{$\begin{array}{c}\text { Validation Cohort } \\
\text { (VHA) }\end{array}$} & \multirow[b]{2}{*}{ Point $^{\mathrm{a}}$} \\
\hline & HR & $P$ Value & HR & $P$ Value & \\
\hline$\underline{\text { Asian race }}$ & 0.26 & $<.01$ & 0.43 & .40 & -3 \\
\hline VTE history & 3.03 & $<.01$ & 4.65 & $<.01$ & +3 \\
\hline Standard dose (120-160 mg) & 1.36 & .05 & 1.18 & .50 & +1 \\
\hline High dose $(>160 \mathrm{mg})$ & 1.58 & .02 & 2.41 & $<.01$ & +2 \\
\hline \multirow[t]{2}{*}{ Risk stratification ${ }^{b}$} & \multicolumn{2}{|c|}{$\mathrm{HR}(\mathrm{high} / \mathrm{low})=1.85(P<.01)$} & \multicolumn{2}{|c|}{$\mathrm{HR}(\mathrm{high} / \mathrm{low})=1.98(P<.01)$} & \multirow[t]{2}{*}{ High if $\geq 2$} \\
\hline & \multicolumn{2}{|c|}{$c$-index $=0.61(95 \% \mathrm{Cl}, 0.57-0.64)$} & \multicolumn{2}{|c|}{ c-index $=0.60(95 \% \mathrm{Cl}, 0.54-0.64)$} & \\
\hline
\end{tabular}

Abbreviations: HR, hazard ratio; VHA, Veterans Health Administration; VTE, venous thromboembolism.

anteger points were assigned according to the $\beta$ coefficients in the model. Variables in the model were aggregated to create the "SAVED" score.

bPatients were stratified to the high-risk group if the score was $\geq 2$ and to the low-risk group if the score was $\leq 1$. For risk stratification, the HR was estimated after regressing the risk group as a binary variable in the Cox regression. Harrell's c-index was estimated after regressing the assigned points as a continuous variable in the Cox regression. The $95 \% \mathrm{Cl}$ for the c-index was estimated from 200 bootstrapped resamples. 


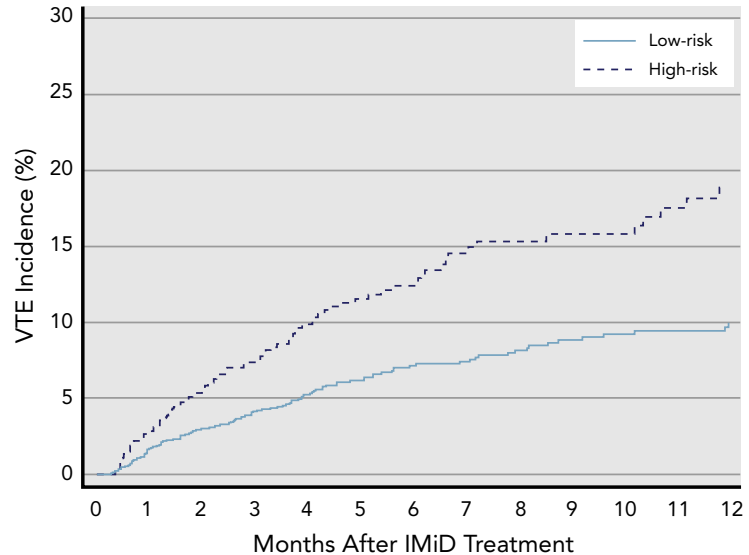

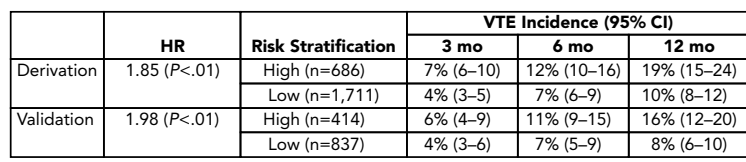

Figure 3. VTE incidence and Kaplan-Meier failure curve of patients stratified by SAVED RAM.

Abbreviations: HR, hazard ratio; IMiD, immunomodulatory drug; RAM, risk assessment model; VTE, venous thromboembolism.

reflecting the contemporary treatment approach to MM. The variables included in the SAVED score are intuitive: history of VTE and recent surgery are well-known risk factors of VTE in the general population, ${ }^{19,20}$ combination regimens with dexamethasone are associated with an increased risk of VTE in $\mathrm{MM},{ }^{2}$ and younger age and Asian race are associated with a lower risk of VTE. ${ }^{19,21,22}$ Conversely, some variables not included in the SAVED model (eg, cytotoxic chemotherapy, LMWH/DOAC use) could impact VTE risk; however, their extremely low proportion $(\sim 1 \%)$ meant that they were not commonly used for first-line treatment in MM. The discrepancy in the association between VTE incidence and age in the derivation and validation datasets may be related to the differential likelihood of receiving subsequent aggressive treatment in the 2 cohorts.

The new RAM represents an improvement to the existing models reported in the MM literature given its early VTE prediction, consistency, and simplicity. It is important to compare this RAM for patients with MM with various existing RAMs for patients with solid tumors. In studies of the latter, the best clinical scores had c-indices of 0.66 to 0.70 in the derivation cohort, ${ }^{23,24}$ although external validations led to lower values of 0.50 to $0.57 .{ }^{25}$ Acquiring additional information on the disease staging and laboratory values might improve the SAVED RAM; however, a recent study showed that the laboratory predictors in the Khorana score used in solid tumors and lymphomas were not predictive for VTE in $\mathrm{MM}^{26}$ By preselecting a cohort of patients with high-risk cancer (MM) and high-risk treatment (IMiD), we inevitably lost some of the key discriminative factors observed in other studies involving a mixture of patients with cancer. Therefore, although we could not predict a true "low-risk" population, a "highest-risk" group of individuals was identified for whom the burden of more aggressive primary anticoagulant thromboprophylaxis rather than aspirin would be warranted at IMiD initiation. This simplified set of clinical risk predictors can serve as the new backbone for further evaluation of novel prognostic biomarkers in this high-risk patient population.

This observational cohort study has several strengths. First, stringent criteria were used to ensure the correct cancer diagnosis, sufficient follow-up, and exclusion of recent VTE. Second, previously validated administrative codes with a high positive predictive value were used to reduce misclassification errors. The observed incidence of VTE in the derivation cohort was similar to the complication rate reported in a contemporary phase III randomized controlled trial of patients with MM (12.5\% at 12 months). ${ }^{27}$ Both UE-DVT and LE-DVT were included in addition to PE for the primary outcome because these were used to characterize thromboembolism in recent VTE prophylaxis trials in patients with cancer. ${ }^{28-30}$ Reassuringly, performance of the SAVED RAM was similar if the outcomes were restricted to only PE and LE-DVT. Fourth, the large number of patients and events allowed for a precise estimation of the VTE risk, and various sensitivity analyses were concordant with the primary results. Finally, despite the use of an independent external validation dataset with different patient demographics and chemotherapy, the derived model had similar discrimination and calibration.

There are also limitations associated with administrative databases. We did not have access to patient-level data, including initial disease staging, laboratory parameters, family history of VTE, or nonprescription medications. The comorbidities captured using claim codes reflected the more severe phenotypes and may have led to the omission of milder cases. We only analyzed the impact of baseline clinical predictors at the time of IMiD initiation and did not assess time-varying risk factors that occurred later in the treatment course. Finally, because of the inherent age limitation of the Medicare database, the RAM was derived from patients aged $>65$ years. Reassuringly, the median age of diagnosis is 67 years for MM, and the RAM retained its significance in the sensitivity analysis that included patients of all ages.

\section{Conclusions}

We derived and externally validated the first VTE RAM for patients with MM starting IMiD therapy. The SAVED model is a parsimonious score with only 5 clinical variables that allows clinicians to readily identify patients at the highest risk of VTE. We are hopeful that this clinical tool will aid informed shared decision-making between providers and patients with MM regarding VTE risk 
before IMiD initiation. Future prospective studies are needed to define the best VTE prevention strategy for patients with MM within a given risk stratum.

\section{Acknowledgments}

The authors acknowledge the efforts of the NCI; the Office of Research, Development and Information, CMS; Information Management Services, Inc.; and the SEER program tumor registries in the creation of the SEER-Medicare database.

Submitted November 8, 2018; accepted for publication January 11, 2019.

Author contributions: Research design: Li, Garcia, Lyman, Sanfilippo.

Statistical analysis: Li, Wu, Luo. Database extraction: Warnick. Data analysis and interpretation: Li, Garcia, Lyman, Sanfilippo. Draft of manuscript: Li. Review and editing of manuscript: Zakai, Libby, Gage.

Disclosures: Dr. Garcia has disclosed that he is a consultant for Janssen. Dr. Lyman has disclosed that he has received grant/research support from Bayer The remaining authors have not received any financial consideration from any person or organization to support the preparation, analysis, results, or discussion of this article.

Funding: Research reported in this publication was supported by National Heart, Lung, and Blood Institute (NHLBl; grants T32HL007093 [Li] and K01HL136893 [Sanfilippo]) and the Conquer Cancer Foundation Young Investigator Award (Li).

Disclaimer: This study used the linked SEER-Medicare database. The interpretation and reporting of these data are the sole responsibility of the authors.

Correspondence: Ang Li, MD, University of Washington, 825 Eastlake Avenue East, M/S CE3-300, Seattle, WA 98109. Email: ali2015@uw.edu

\section{References}

1. Blom JW, Doggen CJM, Osanto S, et al. Malignancies, prothrombotic mutations, and the risk of venous thrombosis. JAMA 2005:293: 715-722.

2. Carrier M, Le Gal G, Tay J, et al. Rates of venous thromboembolism in multiple myeloma patients undergoing immunomodulatory therapy with thalidomide or lenalidomide: a systematic review and meta-analysis. J Thromb Haemost 2011:9:653-663.

3. Palumbo A, Rajkumar SV, Dimopoulos MA, et al. Prevention of thalidomideand lenalidomide-associated thrombosis in myeloma. Leukemia 2008;22: 414-423.

4. Streiff MB, Holmstrom B, Angelini D, et al. NCCN Clinical Practice Guidelines in Oncology: Cancer-Associated Venous Thromboembolic Disease. Version 1.2019. Accessed April 20, 2019. To view the most recent version, visit NCCN.org.

5. Dede RJ, Pruemer JM. Comparing venous thromboembolism prophylactic strategies for ambulatory multiple myeloma patients on immunomodulatory drug therapy. J Oncol Pharm Pract 2016;22: 248-255.

6. Palmaro A, Rougé-Bugat ME, Gauthier M, et al. Real-life practices for preventing venous thromboembolism in multiple myeloma patients: a cohort study from the French health insurance database. Pharmacoepidemiol Drug Saf 2017;26:578-586.

7. Gerds TA, Cai T, Schumacher M. The performance of risk prediction models. Biom J 2008;50:457-479.

8. Royston P, Altman DG. External validation of a Cox prognostic model: principles and methods. BMC Med Res Methodol 2013;13:33.

9. Warren JL, Klabunde CN, Schrag D, et al. Overview of the SEER-Medicare data: content, research applications, and generalizability to the United States elderly population. Med Care 2002;40(Suppl 8):IV-3-18.

10. Lyman $\mathrm{GH}$, Eckert L, Wang $Y$, et al. Venous thromboembolism risk in patients with cancer receiving chemotherapy: a real-world analysis. Oncologist 2013;18:1321-1329.

11. White RH, Garcia M, Sadeghi B, et al. Evaluation of the predictive value of ICD-9-CM coded administrative data for venous thromboembolism in the United States. Thromb Res 2010;126:61-67.

12. National Cancer Institute. $\mathrm{NCl}$ comorbidity index overview. Available at: https://healthcaredelivery.cancer.gov/seermedicare/considerations/ comorbidity.html. Accessed May 15, 2018.

13. Elixhauser A, Steiner C, Harris DR, et al. Comorbidity measures for use with administrative data. Med Care 1998;36:8-27.

14. Chrischilles EA, Winiecki SK, Carnahan RM, et al. Thromboembolic events after immunoglobulin administration. Available at: https://www.sentinelinitiative.org/ vaccines-blood-biologics/assessments/thromboembolic-events-afterimmunoglobulin-administration. Accessed May 15, 2018.

15. Barell V, Aharonson-Daniel L, Fingerhut LA, et al. An introduction to the Barell body region by nature of injury diagnosis matrix. Inj Prev 2002; 8:91-96.
16. Cancer Research Network. Cancer therapy look-up tables. Available at: http://www.hcsrn.org/crn/en/RESEARCH/LookupTables/. Accessed May 15, 2018.

17. Harrell FE Jr, Lee KL, Mark DB. Multivariable prognostic models: issues in developing models, evaluating assumptions and adequacy, and measuring and reducing errors. Stat Med 1996;15:361-387.

18. National Cancer Institute. Number of cancer cases enrolled in Part D. Available at: https://healthcaredelivery.cancer.gov/seermedicare/aboutdata/ enrollees.html. Accessed May 15, 2018.

19. White RH, Zhou H, Romano PS. Incidence of symptomatic venous thromboembolism after different elective or urgent surgical procedures. Thromb Haemost 2003;90:446-455.

20. Prandoni $P$, Lensing $A W$, Cogo $A$, et al. The long-term clinical course of acute deep venous thrombosis. Ann Intern Med 1996;125:1-7.

21. Tsai AW, Cushman M, Rosamond WD, et al. Cardiovascular risk factors and venous thromboembolism incidence: the longitudinal investigation of thromboembolism etiology. Arch Intern Med 2002; 162:1182-1189.

22. Stein PD, Kayali F, Olson RE, et al. Pulmonary thromboembolism in Asians/ Pacific Islanders in the United States: analysis of data from the National Hospital Discharge Survey and the United States Bureau of the Census. Am J Med 2004;116:435-442.

23. Pabinger I, van Es $N$, Heinze $G$, et al. A clinical prediction model for cancer-associated venous thromboembolism: a development and validation study in two independent prospective cohorts. Lancet Haematol 2018;5:e289-298.

24. Khorana AA, Kuderer NM, Culakova E, et al. Development and validation of a predictive model for chemotherapy-associated thrombosis. Blood 2008;111:4902-4907.

25. van Es N, Di Nisio M, Cesarman G, et al. Comparison of risk prediction scores for venous thromboembolism in cancer patients: a prospective cohort study. Haematologica 2017;102:1494-1501.

26. Sanfilippo KM, Wang TF, Luo $S$, et al. Predictive ability of the Khorana score for venous thromboembolism (VTE) in multiple myeloma (MM) [abstract]. J Clin Oncol 2018;36(Suppl 15):Abstract e18733.

27. Bradbury CA, Jenner MW, Striha A, et al. Thrombotic events in patients with myeloma treated with immunomodulatory drugs; results of the myeloma XI study [abstract]. Blood 2017;130(Suppl 1):Abstract 553

28. Carrier M, Abou-Nassar K, Mallick R, et al. Apixaban to prevent venous thromboembolism in patients with cancer. N Engl J Med 2018;380: 711-719.

29. Khorana AA, Francis CW, Kuderer NM, et al. Dalteparin thromboprophylaxis in cancer patients at high risk for venous thromboembolism: a randomized trial. Thromb Res 2017;151:89-95.

30. Khorana AA, Soff GA, Kakkar AK, et al. Rivaroxaban for thromboprophylaxis in high-risk ambulatory patients with cancer. N Engl J Med 2019; 380:720-728. 
Supplemental online content for:

\section{Derivation and Validation of a Risk Assessment Model for Immunomodulatory Drug-Associated Thrombosis Among Patients With Multiple Myeloma}

Ang Li, MD; Qian Wu, PhD; Suhong Luo, MS; Greg S. Warnick; Neil A. Zakai, MD; Edward N. Libby, MD; Brian F. Gage, MD, MSc; David A. Garcia, MD; Gary H. Lyman, MD, MPH; and Kristen M. Sanfilippo, MD, MPHS

J Natl Compr Canc Netw 2019;17(7):840-847

eTable 1: Administrative Claims Codes Search Matrix

eTable 2: Administrative Claims Codes: Outcomes and Comorbidities

eTable 3: Administrative Claims Codes for Medications and Chemotherapy

eTable 4: Univariate Analysis of Risk Factors Associated With VTE in Derivation Cohort

eTable 5: Comparison of Cohorts in the SEER-Medicare Database 
eTable 1. Administrative Claims Codes Search Matrix

\begin{tabular}{|c|c|c|c|c|c|}
\hline & ICD-9-CM Diagnosis Codes & ICD-9-CM Procedure Codes & CPT4/HCPCS & NDC & Comment \\
\hline $\mathrm{NCH}$ & $\begin{array}{l}\text { PDGNS_CD } \\
\text { DGN_CD1-DGN_CD12 } \\
\text { LINEDIAG }\end{array}$ & & HCPCS & & Drop denied claims \\
\hline PDE & & & & GNN & \\
\hline
\end{tabular}

Abbreviations: CPT4, Current Procedural Terminology, 4th edition; DME, durable medical equipment; HCPCS, Healthcare Common Procedure Coding System; MEDPAR, Medicare Provider Analysis and Review; NCH, National Claims History (carrier claims); NDC, National Drug Code; OUTPAT, outpatient claims;

PDE, Medicare Part D Prescription Drug Event; SNF, skilled nursing facility; VTE, venous thromboembolism. 
2 - Li et al

\section{eTable 2. Administrative Claims Codes: Outcomes and Comorbidities}

VTE outcome (day 7+ look-forward window) $)^{1,2}$

Pulmonary embolism

ICD-9-CM diagnosis: 415.11, 415.12, 415.13, 415.19

Deep vein thrombosis

ICD-9-CM diagnosis: 453.2, 453.40, 453.41, 453.42, 453.8, 453.82, 453.83, 453.84, 453.85, 453.86, 453.87, 453.89, 453.9

Phlebitis

ICD-9-CM diagnosis: 451.11, 451.19, 451.2, 451.81, 451.83, 451.84, 451.89, 451.9

$\mathrm{NCl}$ Comorbidity Index (365-day lookback window) ${ }^{3}$

Myocardial infarction

ICD-9-CM diagnosis: $410 . x x$ with inpatient length of stay $>2$ d; ICD-9-CM diagnosis: $412 . b b$

Congestive heart failure

ICD-9-CM diagnosis: 398.91, 425.4x-425.5x, 425.7x-425.9x, 428.xx

Paralysis/Immobilization

ICD-9-CM diagnosis: 342.xx, 344.0x-344.6x, 344.9x

Diabetes

ICD-9-CM diagnosis: 250.bb, 250.0x-250.3x, 250.4x-250.9x, 362.0x

Renal disease

ICD-9-CM diagnosis: 403.01, 403.11, 403.91, 404.02, 404.03, 404.12, 404.13, 404.92, 404.93, 582.xx-583.xx, 585.xx-586.xx, 588.xx, V42.0x, V45.1x, V56.xx ICD-9-CM procedure: $39.27,39.42,39.95,54.98,55.69$

Liver disease

ICD-9-CM diagnosis: 070.22-070.23, 070.32-070.33, 070.44, 070.54, 456.0x-456.2x, 571.2x, 571.4x-571.6x, 572.2x-572.8x, V42.7x

ICD-9-CM procedure: $39.1 \mathrm{~b}, 42.91,50.5 \mathrm{x}$

Elixhauser comorbidity index (365-day lookback window) ${ }^{4}$

Cardiac arrhythmias

ICD-9-CM diagnosis: 426.10, 426.11, 426.13, 426.2-426.53, 426.6-426.8, 427.0, 427.2, 427.31, 427.60, 427.9, 785.0, V45.0, V53.3

Hypertension

ICD-9-CM diagnosis: 401.1, 401.9, 402.10, 402.90, 404.10, 404.90, 405.1, 405.9

Coagulopathy

ICD-9-CM diagnosis: 286.x, 287.1, 287.3-287.5

Anemia

ICD-9-CM diagnosis: 280.0, 280.1-281.9, 285.9

Obesity

ICD-9-CM diagnosis: 278.0

VTE-specific risk factors (365-day lookback window unless stated)

Central venous catheter ${ }^{5}$

ICD-9-CM diagnosis: 38.97

CPT4: 36555-36558, 36560-36561, 36563, 36565-36566, 36568-36571, 36575-36576, 36578, 36580-36585, 36597-36598

Sepsis (90-day lookback window $\left.{ }^{5}\right)$

ICD-9-CM diagnosis: 003.1, 020.2, 022.3, 036.2, 038.x, 054.5, 449, 785.52, 785.5x, 790.7, 995.91, 995.92

(continued on next page)

Abbreviations: CPT4, Current Procedural Terminology, version 4; HCPCS, Healthcare Common Procedure Coding System; VTE, venous thromboembolism. 


\section{eTable 2. Administrative Claims Codes: Outcomes and Comorbidities (cont.)}

VTE-specific risk factors (365-day lookback window unless stated) (cont.)

Surgery (90-day lookback window $)^{5}$ :

ICD-9-CM diagnosis: V67.0x

ICD-9-CM procedure: $00.7 x, 00.8 x, 03.4,03.5 x, 03.9 x, 79.85,79.86,80.45,80.46,80.6,80.85,80.86,80.95,80.96,81.40,81.42,81.43,81.51,81.52,81.53$, $81.54,81.55,84.16,84.18$

CPT4: 01214-01215, 01402, 20930-20938, 22010-22015, 22100-22116, 22206-22226, 22318-22328, 22532-22534, 22548-22585, 22590-22632,

22800-22819, 22830, 22840-22865, 27075-27079, 27130-27138, 27218, 27226-27228, 27253, 27258-27259, 27299, 27447, 27486-27487, 29861-29863,

43644-43645, 43800-43881, 49570-49575, 50010-50045, 50070, 50100-50135, 50205-50290, 50320-50340, 50370, 50382-50384, 50400-50540, 50593,

50600-50630, 50650-50660, 50700-50940, 51020-51040, 51080, 53000-53085, 53210-53275, 53400-53520, 53855, 56620-56740, 56800-56810,

57000-57335, 57530-57556, 57720, 58140-58146, 58150-58294, 58400-58540, 58600-58615, 58700-58720, 58750-58770, 58820-58825, 58920-58960,

$58999,61320-61321,61546,61680-61692,61697-61710,62160-62165,63001-63017,63045-63051,63055-63066,63075-63091,63101-63103$, 63170-63200, 63250-63295, 63300-63308, 63650-63688

HCPCS: S2083, S2213

Traumatic injury (90-day lookback window $\left.{ }^{6}\right)$ :

ICD-9-CM diagnosis: 800-959, except 905-909, 930-939

Personal history of VTE (183- to 365-day lookback window)

ICD-9-CM diagnosis: V12.51, V12.52, V12.55; 415.x, 451.x, 453.x

Personal history of bleeding ${ }^{7}$

ICD-9-CM diagnosis: $423.00,430.00,431.00,432.00,432.1,432.9,455.20,455.50,455.80,459.00,456.00,456.20,530.70,530.80,531.00,531.01,531.20$, $531.21,531.40,531.41,531.60,531.61,532.00,532.01,532.20,532.21,532.40,532.41,532.60,532.61,533.00,533.01,533.20,533.21,533.40,533.41$, $533.60,533.61,534.00,534.01,534.20,534.21,534.40,534.41,534.60,534.61,535.01,535.11,535.21,535.31,535.41,535.51,535.61,537.80,562.02$, $562.03,562.12,568.81,569.30,569.85,578.00,578.10,578.90,593.81,599.70,623.80,626.20,626.60,719.10,719.11,719.12,719.13,719.14,719.15$, $719.16,719.17,719.18,784.70,784.80,786.30$

Abbreviations: CPT4, Current Procedural Terminology, version 4; HCPCS, Healthcare Common Procedure Coding System; VTE, venous thromboembolism.

\section{References}

1. Lyman GH, Eckert L, Wang Y, et al. Venous thromboembolism risk in patients with cancer receiving chemotherapy: a real-world analysis. Oncologist 2013;18: $1321-1329$.

2. White RH, Garcia M, Sadeghi B, et al. Evaluation of the predictive value of ICD-9-CM coded administrative data for venous thromboembolism in the United States. Thromb Res 2010;126:61-67.

3. National Cancer Institute. NCl comorbidity index overview. Available at: https://healthcaredelivery.cancer.gov/seermedicare/considerations/comorbidity.html. Accessed May 15, 2018.

4. Elixhauser A, Steiner C, Harris DR, et al. Comorbidity measures for use with administrative data. Med Care 1998;36:8-27.

5. Chrischilles EA, Winiecki SK, Carnahan RM, et al. Thromboembolic events after immunoglobulin administration. Available at: https://www.sentinelinitiative.org/ vaccines-blood-biologics/assessments/thromboembolic-events-after-immunoglobulin-administration. Accessed May 15, 2018.

6. Barell V, Aharonson-Daniel L, Fingerhut LA, et al. An introduction to the Barell body region by nature of injury diagnosis matrix. Inj Prev 2002;8:91-96.

7. Arnason T, Wells PS, van Walraven C, et al. Accuracy of coding for possible warfarin complications in hospital discharge abstracts. Thromb Res 2006;118:253-262. 


\section{4 - Li et al}

\section{eTable 3. Administrative Claims Codes for Medications and Chemotherapy}

Concurrent chemotherapy $\pm 30 \mathrm{~d}^{1}$

ICD-9 procedure: injection/infusion chemotherapy/immunotherapy $(99.25,99.28)$

CPT4: injection/infusion chemotherapy (96401-96417), intra-arterial chemotherapy (96420-96425), other injection/infusion chemotherapy (96440-96549) NDC: melphalan, cyclophosphamide, etoposide

HCPCS: chemotherapy infusion (Q0083-Q0085), melphalan (J8600, J9245), bendamustine (J9033, J9034, C9243), vincristine (J9370, J9371, J9375, J9380), cyclophosphamide (J8530, J9070, J9080, J9090, J9091-J9097, C9420, C9421), etoposide (J8560, J9181, J9182, C9414, C9425), carboplatin (J9045), cisplatin (J9060, J9062, C9418), mitoxantrone (J9293), daunorubicin (J9150, J9151, C9424), idarubicin (J9211, C9429), doxorubicin (J9000, J9001, J9002, Q2048, Q2049, Q2050, C9415), bortezomib (J9041, C9207)

Concurrent erythropoietin $\pm 30 \mathrm{~d}^{1}$

GNN: darbepoetin alfa, epoetin alfa

NDC: darbepoetin, epoetin

HCPCS: Q4081, J0881, J0882, J0885, J0886

Concurrent dexamethasone $\pm 30 \mathrm{~d}$

GNN: dexamethasone

Concurrent anticoagulant $<30 \mathrm{~d}$ to $>7 \mathrm{~d}$

GNN: enoxaparin, dalteparin, tinzaparin, fondaparinux, heparin, warfarin, dabigatran, rivaroxaban, apixaban, edoxaban

Abbreviations: CPT4, Current Procedural Terminology, version 4; GNN, generic drug name; HCPCS, Healthcare Common Procedure Coding System; NDC, National Drug Code.

\section{Reference}

1. Cancer Research Network. Cancer therapy look-up tables (U24 CA171524). Available at: http://www.hcsrn.org/crn/en/RESEARCH/LookupTables/. Accessed May 15, 2018. Last updated March 2018. 


\section{eTable 4. Univariate Analysis of Risk Factors Associated With VTE in Derivation Cohort}

\begin{tabular}{|c|c|c|}
\hline & HR & $P$ Value \\
\hline \multicolumn{3}{|l|}{ NCCN risk factors } \\
\hline Prior VTE & 3.20 & $<.01$ \\
\hline Obesity & 0.86 & .63 \\
\hline Central venous catheter or pacemaker & 0.75 & .68 \\
\hline Cardiac disease $(\mathrm{CHF}, \mathrm{Ml}, \mathrm{CA})$ & 1.00 & .98 \\
\hline Chronic renal disease & 1.14 & .38 \\
\hline Diabetes & 1.14 & .38 \\
\hline Acute infection & 1.42 & .20 \\
\hline Immobilization & 1.24 & .71 \\
\hline General surgery or anesthesia & 1.82 & .12 \\
\hline Trauma & 0.96 & .83 \\
\hline Erythropoietin & 0.89 & .61 \\
\hline Blood-clotting disorders & 0.96 & .86 \\
\hline High-dose dexamethasone, $\geq 480 \mathrm{mg}$ & 1.09 & .55 \\
\hline Doxorubicina $^{a}$ & NE & N/A \\
\hline Multiagent (cytotoxic) chemotherapy & 2.05 & .48 \\
\hline \multicolumn{3}{|l|}{ Additional risk factors considered } \\
\hline Age (every 10-y increase) & 1.23 & .04 \\
\hline Sex (female vs male) & 1.21 & .16 \\
\hline \multicolumn{3}{|l|}{ Race } \\
\hline White & Ref & \\
\hline Black & 1.33 & .13 \\
\hline Asian & 0.25 & $<.01$ \\
\hline Year of diagnosis (every 1-y increase) & 0.98 & .66 \\
\hline Geography (metropolitan vs rural) & 0.92 & .67 \\
\hline Liver disease & 1.15 & .81 \\
\hline Anemia & 0.93 & .58 \\
\hline Hypertension & 1.28 & .10 \\
\hline History of bleeding & 1.22 & .41 \\
\hline IMiD type (lenalidomide vs thalidomide) & 1.13 & .47 \\
\hline \multicolumn{3}{|l|}{ Concurrent chemotherapy } \\
\hline Bortezomib & 0.85 & .37 \\
\hline Melphalan & 1.72 & .45 \\
\hline Cyclophosphamide & 1.18 & .77 \\
\hline
\end{tabular}

(continued) Abbreviations: $\mathrm{CA}$, cardiac arrhythmia; $\mathrm{CHF}$, congestive heart failure; $\mathrm{HR}$, hazard ratio; IMiD, immunomodulatory drug; MI, myocardial infarction; N/A, not applicable; NE, no event; VTE, venous thromboembolism.

aHR was not estimable for this variable because there was no VTE event in the comparison group.

bDexamethasone was reorganized into modern conventional standard and high-dose categories.

\section{eTable 4. Univariate Analysis of Risk Factors Associated With VTE in Derivation Cohort (cont.)}

\begin{tabular}{|c|c|c|}
\hline & HR & $P$ Value \\
\hline \multicolumn{3}{|l|}{ Additional risk factors considered (cont.) } \\
\hline \multicolumn{3}{|l|}{ Concurrent dexamethasone ${ }^{b}$} \\
\hline None or low dose $(<120 \mathrm{mg} /$ cycle $)$ & Ref & \\
\hline Standard dose (120-160 mg/cycle) & 1.27 & .13 \\
\hline High dose (>160 mg/cycle) & 1.42 & .06 \\
\hline \multicolumn{3}{|l|}{ Concurrent anticoagulation } \\
\hline None & Ref & \\
\hline Warfarin & 1.09 & .69 \\
\hline Low-molecular-weight heparin & 0.38 & .34 \\
\hline Direct oral anticoagulant ${ }^{a}$ & NE & N/A \\
\hline
\end{tabular}

Abbreviations: $\mathrm{CA}$, cardiac arrhythmia; $\mathrm{CHF}$, congestive heart failure; $H R$, hazard ratio; IMiD, immunomodulatory drug; MI, myocardial infarction; N/A, not applicable; NE, no event; VTE, venous thromboembolism.

aHR was not estimable for this variable because there was no VTE event in the comparison group.

bDexamethasone was reorganized into modern conventional standard and high-dose categories. 


\section{eTable 5. Comparison of Cohorts in SEER-}

Medicare Database

\begin{tabular}{|lcc|}
\hline & $\begin{array}{c}\text { IMiD Cohort } \\
(\mathbf{N = 2 , 3 9 7 )} \\
\%(\mathbf{n})\end{array}$ & $\begin{array}{c}\text { MM Cohort } \\
\mathbf{( N = 3 0 , 7 5 7 )} \\
\%(\mathbf{n})\end{array}$ \\
\hline $\begin{array}{c}\text { Age, y } \\
<65\end{array}$ & 0 & $26 \%(7,873)$ \\
\hline $65-69$ & $25 \%(592)$ & $17 \%(5,274)$ \\
\hline $70-74$ & $28 \%(671)$ & $17 \%(5,247)$ \\
\hline $75-79$ & $23 \%(542)$ & $16 \%(4,917)$ \\
\hline $80-84$ & $15 \%(360)$ & $13 \%(4,078)$ \\
\hline$\geq 85$ & $10 \%(232)$ & $11 \%(3,368)$ \\
\hline Sex & & \\
\hline Male & $51 \%(1,216)$ & $56 \%(17,161)$ \\
\hline Female & $49 \%(1,181)$ & $44 \%(13,596)$ \\
\hline
\end{tabular}

Race

\begin{tabular}{lcc}
\hline White & $80 \%(1,909)$ & $75 \%(22,987)$ \\
\hline Black & $13 \%(303)$ & $19 \%(5,878)$ \\
\hline Asian/Pacific Islander & $7 \%(146)$ & $5 \%(1,486)$ \\
\hline $\begin{array}{l}\text { American Indian or } \\
\text { Alaskan Native }\end{array}$ & $0.5 \%(13)$ & $0.4 \%(137)$ \\
\hline Unknown/Missing & $1 \%(26)$ & $1 \%(269)$
\end{tabular}

Year of diagnosis

\begin{tabular}{lcc}
\hline$<2007$ & 0 & $3 \%(1,072)$ \\
\hline 2007 & $8 \%(202)$ & $13 \%(3,904)$ \\
\hline 2008 & $13 \%(311)$ & $14 \%(4,142)$ \\
\hline 2009 & $12 \%(294)$ & $14 \%(4,237)$ \\
\hline 2010 & $14 \%(341)$ & $14 \%(4,346)$ \\
\hline 2011 & $15 \%(362)$ & $14 \%(4,358)$ \\
\hline 2012 & $18 \%(419)$ & $14 \%(4,401)$ \\
\hline 2013 & $20 \%(468)$ & $14 \%(4,297)$
\end{tabular}

\begin{tabular}{lcc}
\hline SEER region & $32 \%(761)$ & $36 \%(11,181)$ \\
\hline California & $5 \%(125)$ & $5 \%(1,524)$ \\
\hline Connecticut & $5 \%(119)$ & $6 \%(1,931)$ \\
\hline Detroit & $13 \%(306)$ & $13 \%(3,907)$ \\
\hline Georgia & $1 \%(25)$ & $1 \%(449)$ \\
\hline Hawaii & $8 \%(180)$ & $4 \%(1,360)$ \\
\hline lowa & $8 \%(180)$ & $6 \%(1,768)$ \\
\hline Kentucky & $6 \%(152)$ & $6 \%(1,875)$ \\
\hline Louisiana & $14 \%(324)$ & $12 \%(3,656)$ \\
\hline New Jersey & $2 \%(50)$ & $2 \%(643)$ \\
\hline New Mexico & $6 \%(138)$ & $6 \%(1,736)$ \\
\hline Seattle & $2 \%(37)$ & $1 \%(727)$ \\
\hline Utah & $87 \%(2,092)$ & $92 \%(28,213)$ \\
\hline Geographic region & $13 \%(305)$ & $8 \%(2,544)$ \\
\hline Metropolitan & & \\
\hline Rural & & \\
\hline
\end{tabular}

Abbreviations: IMiD, immunomodulatory drug; MM, multiple myeloma. 